\title{
Signatures of positive selection in the cis-regulatory sequences of the human oxytocin receptor (OXTR) and arginine vasopressin receptor 1a (AVPR1A) genes
}

Helmut Schaschl ${ }^{1}$, Susanne Huber ${ }^{1}$, Katrin Schaefer ${ }^{1}$, Sonja Windhager ${ }^{1}$, Bernard Wallner ${ }^{1,2,3}$ and Martin Fieder ${ }^{{ }^{*}}$

\begin{abstract}
Background: The evolutionary highly conserved neurohypophyseal hormones oxytocin and arginine vasopressin play key roles in regulating social cognition and behaviours. The effects of these two peptides are meditated by their specific receptors, which are encoded by the oxytocin receptor (OXTR) and arginine vasopressin receptor 1a genes (AVPRTA), respectively. In several species, polymorphisms in these genes have been linked to various behavioural traits. Little, however, is known about whether positive selection acts on sequence variants in genes influencing variation in human behaviours.

Results: We identified, in both neuroreceptor genes, signatures of balancing selection in the cis-regulative acting sequences such as transcription factor binding and enhancer sequences, as well as in a transcriptional repressor sequence motif. Additionally, in the intron 3 of the OXTR gene, the SNP rs59190448 appears to be under positive directional selection. For rs59190448, only one phenotypical association is known so far, but it is in high LD' $(>0.8)$ with loci of known association; i.e., variants associated with key pro-social behaviours and mental disorders in humans.

Conclusions: Only for one SNP on the OXTR gene (rs59190448) was a sign of positive directional selection detected with all three methods of selection detection. For rs59190448, however, only one phenotypical association is known, but rs59190448 is in high LD' (>0.8), with variants associated with important pro-social behaviours and mental disorders in humans. We also detected various signatures of balancing selection on both neuroreceptor genes.
\end{abstract}

Keywords: Oxytocin receptor, Arginine vasopressin 1a receptor, Positive selection, Balancing selection, Genetic diversity

\section{Background}

Human behaviours are characterised by marked differences on both the population and individual level. The two evolutionary-conserved neurohypophyseal hormones oxytocin and arginine vasopressin and their receptors play key roles in modulating cognition and social behaviours in mammals [1]. The corresponding genes are expressed mainly in the hypothalamus and secreted from the posterior pituitary gland. The main hormonal activity of oxytocin is to regulate parturition and lactation. Vasopressin, in contrast, regulates salt

\footnotetext{
* Correspondence: martin.fieder@univie.ac.at

'Department of Anthropology, University of Vienna, Vienna, Austria

Full list of author information is available at the end of the article
}

and water homeostasis. The neurophysiological effects of oxytocin are shown in facilitating social communication, affiliative behaviours, and social cognition. Accordingly, oxytocin has been reported to influence maternal behaviour, trust, generosity, empathy, eye contact, face memory, and also to reduce anxiety [2]. Vasopressin expressed in the brain modulates social behaviours in sexually dimorphic ways, including anxietyrelated behaviour as shown in animal studies, and may act to enhance social recognition and the emotional response to stress [3-5]. Similar effects have also been reported in humans, especially with regard to aggression and the perception of anger [6]. For instance, cerebrospinal fluid levels of vasopressin are directly correlated 
with general aggression and aggression against persons [7]. Vasopressin may influence aggression in males by priming individuals to respond to emotionally ambiguous social stimuli as if they were threatening or aggressive [8]. Additionally, genetic variation within the AVPR1A gene, particular in the $5^{\prime}$ upstream region (5' UTR) in both humans and chimpanzees, has been linked to relationship quality and pair bonding [9], personality traits $[1,10,11]$, and autism disorders $[12,13]$. In particular, polymorphic microsatellites in the $5^{\prime}$ flanking promoter region have been associated with species-specific and individual variation in social behavior in mice [14]. Furthermore, two SNPs (rs7294536 and rs10877969) have been identified in the promoter region of the human AVPR1A gene, which were significantly associated with autism in Korean subjects [13].

The physiological effects of these two neuroactive peptides are mediated via their specific receptor molecules, encoded by the oxytocin receptor gene (OXTR) and arginine vasopressin receptor 1a gene (AVPR1A). These two genes share a feature unique to the $G$ proteincoupled receptor genes: an intron located before the seventh transmembrane domain of the receptor encoding sequences [1]. In several species, polymorphisms in these genes, mainly in the non-coding regions, have been linked to inter-individual variation in cognition and social behaviours $[1,15]$.

Recently, it has been reported that polymorphisms in the OXTR gene are associated with the development of psychopathy, for example in children with conduct problems or with callous and unemotional traits [16]. Intronic SNPs on OXTR are further associated with autistic spectrum disorder (ASD). Nonetheless. alleles appear to have opposite effects in Asian compared to Caucasian populations [17-19]. Polymorphisms in and near the 3'UTR is also involved in ASD risk [9,20,21]. A recent meta-analysis including 3941 individuals from 11 independent samples and 16 SNPs on OXTR, however, found a significant association with ASD for only 4 SNPs (i.e., rs7632287, rs237887, rs2268491, rs2254298) [22]. In addition, specific sequence variants of OXTR are associated with the Asperger Syndrome [23]. Finally, an allelic variation of rs53576 on OXTR apparently influences human empathy [24], and various SNP variations are associated with maternal sensitivity [25] and parental touch provision [26]; rs7632287 on OXTR plays also a role in pair-bonding [27].

Additionally, support seeking is associated with rs53576 on OXTR as well, but again different in Caucasian Americans compared with Koreans [28]. Rs53576 is also associated with pro-sociality: Kogan et al. [29] reported that individuals homozygous for the $G$ allele on rs53576 were rated more pro-social than carriers of the A allele. Moreover, rs53576 influences trust behaviour
[30], stress reactivity [24], and lower self- reported affect [31]. There is also evidence that OXTR SNPs (rs2228485, rs53576) help process social information from the emotion expression of faces and the voice [24,31].

In economic games, SNPs on OXTR play a role, too: Israel et al. [32] demonstrated that the SNPs rs1042778, rs 2268490 , and rs 237887 on OXTR intron 3 are associated with performance in the dictator game and the social value task. Although this association also holds true in an independent female sample [32], in a Swedish population (performing the dictator and the trust-game) neither associations reported by Isreal et al. [32], nor any association with rs53576, could be confirmed. Extensive reviews of OXTR associations can be found in [1] and [33].

Thus, oxytocin and its specific receptor are crucial for a range of social behaviours and disorders associated with social dis-functioning. Moreover, oxytocin-related cultural differences in social behaviour exist among different human populations [28].

In summary, these studies suggest that genetic variation, particularly in the non-coding DNA, of both neuroreceptor genes significantly influences social behaviour in humans. Little, however, is known about whether positive selection may have shaped sequence variation at loci involved in social cognition. In most studies, genes that show signatures of positive selection usually include loci involved in hostpathogen interactions, reproduction, dietary adaptation, and skin and hair coloration [34].

Only two studies have so far reported positive selection for genes involved in human behaviours. Ding et al. [35] suggested that the seven-repeat variant of the dopamine receptor gene D4 (DRD4 7R allele) originated as a rare mutational event, but increased to high frequency in human populations due to positive selection (but see [36]). The allele DRD4 7R is reportedly associated with certain psychiatric phenotypes such as the attention-deficit hyperactivity disorder (ADHD) [37,38]. The other study reported an excess of high frequency-derived haplotypes for the monoamine oxidase A gene (MAO-A), perhaps reflecting recent positive selection [39]. This gene shows association with borderline mental retardations, depression, and psychopathy [40-42].

In the present study, we thus examined whether positive selection has shaped the polymorphism in the OXTR and AVPR1A genes. We utilized large SNP datasets released by the 1000 Genomes project (http:// www.1000genomes.org/) [43]. We used the degree of genetic differentiation (measured by $F_{S T}$ ) over many worldwide populations as a proxy for positive selection at the SNP level. This approach enables detecting the signature of recent positive selection in human populations. We further investigated whether any SNPs identified under selection are known to be associated with human behavioural traits. 


\section{Results}

\section{Regression trees}

The OXTR gene sample of the 14 analysed human populations from the 1000 Genomes project is separated by nine SNPs, dividing the tree consecutively (rs59190448, rs1170 6648, rs9840864, rs34992398, rs7610471, rs2139184, rs237 916, rs35498753, rs237902). Starting with rs59190448, the tree unfolds from node to node (SNPs), dividing the populations as shown in Fig. 1. In the AVPR1a gene, the seven SNPS (rs3759292, rs7980289, rs34310119, rs11174816, rs11174817, 11174818, rs182223193) unfold the regression tree (Fig. 4).

\section{Detecting selection in the OXTR gene}

All three approaches (FDIST, BayeScan and the extended Lewontin and Krakauer test (FLK)) identified the SNP rs59190448 (in the intron 3) to be significantly under positive directional selection (Table 1). Furthermore, FDIST and BayeScan detected the SNP rs2268494 in the OXTR gene under balancing selection (Table 1, Fig. 2 and Fig. 3, Additional file 1: Table S1).

The SNP rs59190448 also unfolds the regression tree (Fig. 1) and is characterised by an $F_{S T}>0.25$. In the populations with African origin (populations ASW, LWK and YRI), rs59190448 appears as the homozygote AA allele (ancestral allele). The heterozygote allele AG is mostly present in African populations and only occasionally in two European populations (CEU, GBR). In all the other populations, rs59190448 is present only as the homozygote GG allele. Interestingly, the Neanderthal genome sequence (Assembly and Analysis tracks on UCSC Genome Browser) is present as the homozygote

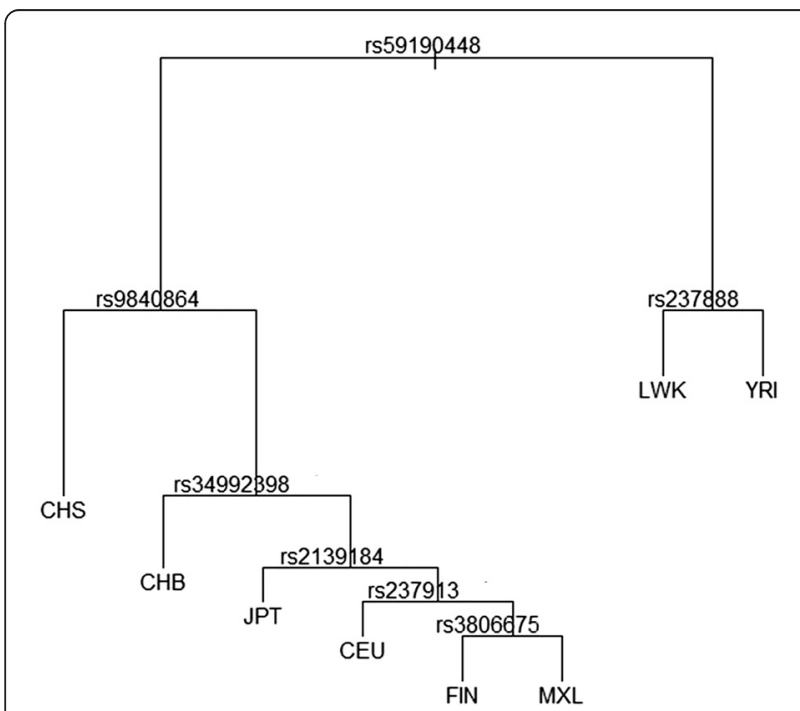

Fig. 1 Regressions tree of the 122 SNPs on OXTR regressing on the 14 human populations from 1000genomes. The regression trees shows the SNPs (nodes) according to which the 14 population do separate from each other
GG allele at this locus, whereas the Denisova genome sequence appears as the homozygote AA allele. From the 1000 Genomes data, we further calculated that rs59190448 is in high LD (>0.8) with an array of SNPs of known social behavioural associations, namely rs237889, rs13316193, rs2254298, and rs1042778.

The SNP rs2268494, which is under balancing selection, is located within a CTCF (zinc finger protein) transcription factor binding sequence. The CTCF protein acts as a transcriptional regulator and is implicated in genomic imprinting. In addition, FDIST detected the upstream gene SNP variants rs4643699 and rs1488467 to be under balancing selection (Table 1, Fig. 2).

\section{Detecting selection in the AVPR1A gene}

Even though some SNPs on the AVPR1A gene unfold the regression tree (Fig. 4), no SNPs were detected under directional selection by the FDIST and BayeScan approaches. The FLK test, however, detected the SNP rs3759292, which is located in the promotor region of AVPR1A, as being under directional selection (Table 2).

This result should be treated with caution because only FLK detected this SNP as being under positive selection. Nonetheless, FDIST and BayScan identified the SNP rs34462214 (in the intron) and the SNP rs7308855 (upstream variant) to be under balancing selection (Table 2, Fig. 4, Fig. 5, Fig. 6). The APVR1A upstream SNP rs7308855 is located within the transposable element hAT-Charlie family DNA transposon (MER103C), which is an element found only in mammals and plays an important role in gene regulation [33]. Furthermore, FDIST also identified several other SNPs as being significantly $(P<0.005)$ under balancing selection. The BayeScan approach detected at the level of $\log 10$ (BF) $1 \rightarrow 1.5$ (i.e., 'strong evidence' for selection), congruent with FDIST, the following SNPs as being under selection: rs12830859, rs11174811, and rs3021529 (Table 2). In Additional file 1: Table S1 and Additional file 1: Table S2, $F_{S T}$ values and detailed results from FDIST and BayeScan for all loci on OXTR, respectively on AVPR1 are displayed.

\section{Discussion}

This study shows that, the OXTR intronic SNP rs2268494 detected as being under balancing selection is associated with reactions to betrayal [44] as well as the autism spectrum disorder (ASD [21]). Interestingly, this SNP is located within a CTCF (zinc finger protein) transcription factor binding motif, which is recognised by the CTCF (CCCTC-binding factor (zinc finger protein)) transcription factor. This transcriptional regulator protein, which can activate or repress transcription, is associated with diseases that include mental retardation [45]. 


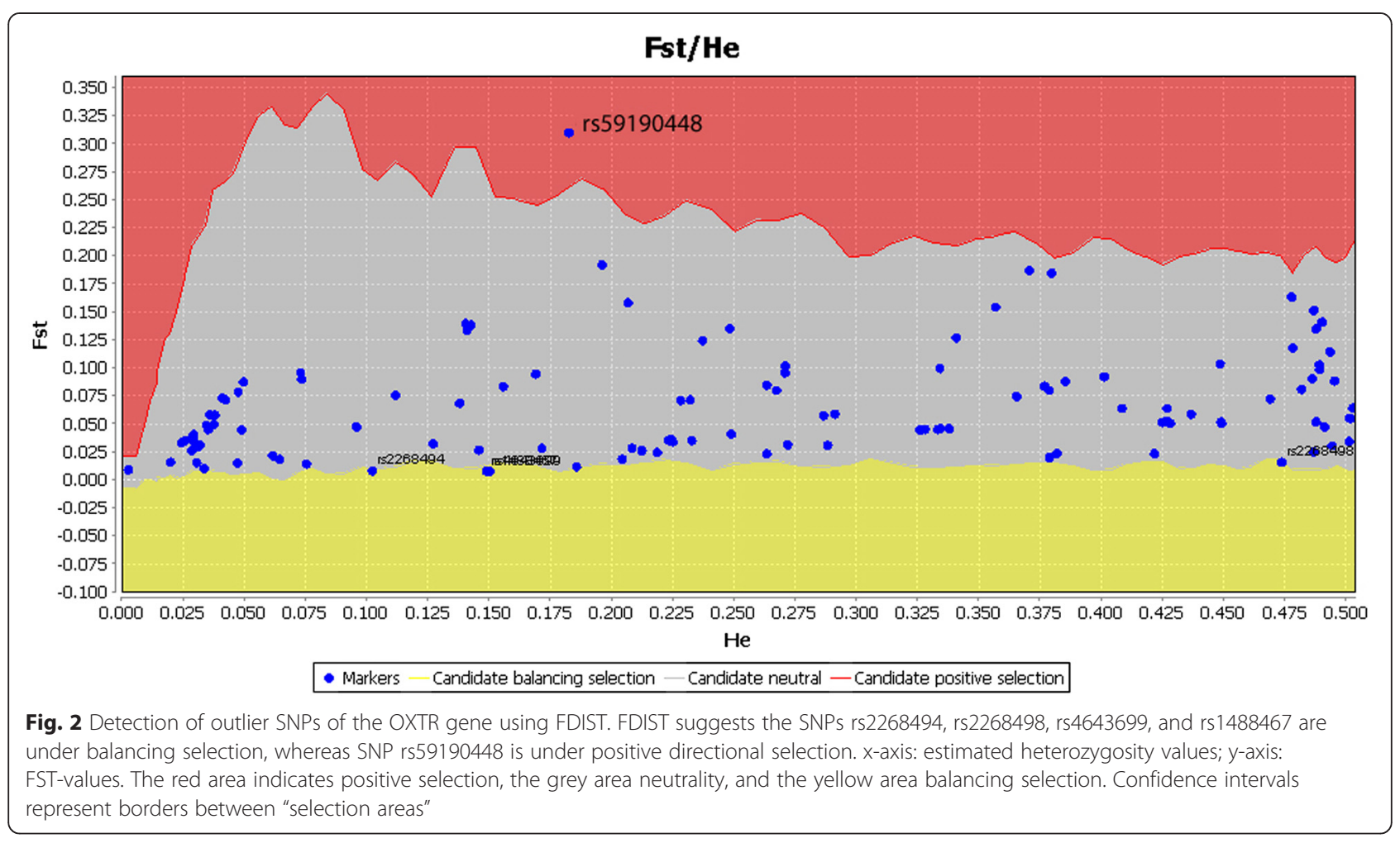

It remains unknown whether this transcription factor influences the gene expression of the OXTR gene.

In the AVPR1A gene, at least two of the three approaches detected the SNPs rs34462214 and rs7308855 as being under balancing selection. For the SNP rs34462214, no phenotypic associations are reported yet. The SNP rs7308855, however, is located in a DNA transposon sequence region (a hAT-Charlie family DNA transposon) found only in placental mammals [46]. This transposon family a) has the epigenetic signatures of enhancers, insulators, and repressors, b) directly binds transcription factors essential for pregnancy, and c) coordinately regulates gene expression in response to progesterone and cAMP [46]. The latter study concluded that this transposable element contributed to the origin of a novel gene regulatory network dedicated to pregnancy in placental mammals.
On AVPR1a the signature of balancing selection could be adaptive because of the pleiotropic function of this gene. In addition, FDIST (as well as BayeScan with $\log 10(\mathrm{BF})$ $1 \rightarrow 1.5$, i.e., 'strong evidence' for selection) detected congruently several more SNPs under selection in the AVPR1A gene. From these SNPs under selection, rs11174811 and rs3021529 are located in important regulatory sequences. The SNP rs11174811 is located in potential microRNA binding sites [35]. Studies suggest that microRNA binding sites in the 3'UTR are potent regulators of gene expression in animals. Indeed, this SNP has been found to be associated with expression levels of the AVPR1A gene in human brain tissue [47]. Interestingly, this locus is associated with risk of drug use disorders in humans [47]. The SNP rs3021529 is located within several transcription factor binding sequences, such as for the enhancer and for the

Table 1 Identified outlier loci in the OXTR gene that are under balancing selection and directionaldirectionalselection using the approaches FDIST, BayeScan and F.LK

\begin{tabular}{|c|c|c|c|c|c|c|c|c|c|c|}
\hline \multirow[b]{2}{*}{ Locus } & \multirow[b]{2}{*}{ Function } & \multicolumn{3}{|l|}{ FDIST } & \multicolumn{3}{|l|}{ BayeScan } & \multicolumn{3}{|c|}{ FLK } \\
\hline & & $H_{E}$ & $F_{S T}$ & $P$ & $\log 10(B F)$ & $q$ & $a_{i}$ & $F_{S T}$ & F.LK & F.LK.p.val \\
\hline rs2268494 & Intron 3 & 0.1022 & 0.0083 & 0.0000 & 1.8492 & 0.0159 & -1.3815 & 0.0291 & & \\
\hline rs59190448 & Intron 3 & 0.1825 & 0.3103 & 0.0004 & 3.3977 & 0.0004 & 1.447 & 0.2548 & 13.521 & 0.001 \\
\hline rs2268498 & Upstream & 0.4736 & 0.0159 & 0.0009 & & & & & & \\
\hline rs4643699 & Upstream & 0.15 & 0.0081 & 0.0000 & & & & & & \\
\hline rs 1488467 & Upstream & 0.1487 & 0.0081 & 0.0000 & & & & & & \\
\hline
\end{tabular}

The results with significance $P$ value $<0.005$ are shown for FDIST and F.LK; ${ }^{*}$ the BayeScan log $10(B F)>1.5$ indicating 'very strong' evidence $(P(a \neq l o=0.03 \rightarrow 0.01)$ for selection. The SNP rs59190448 is under directionalselection and the remaining SNPs under balancing selection 


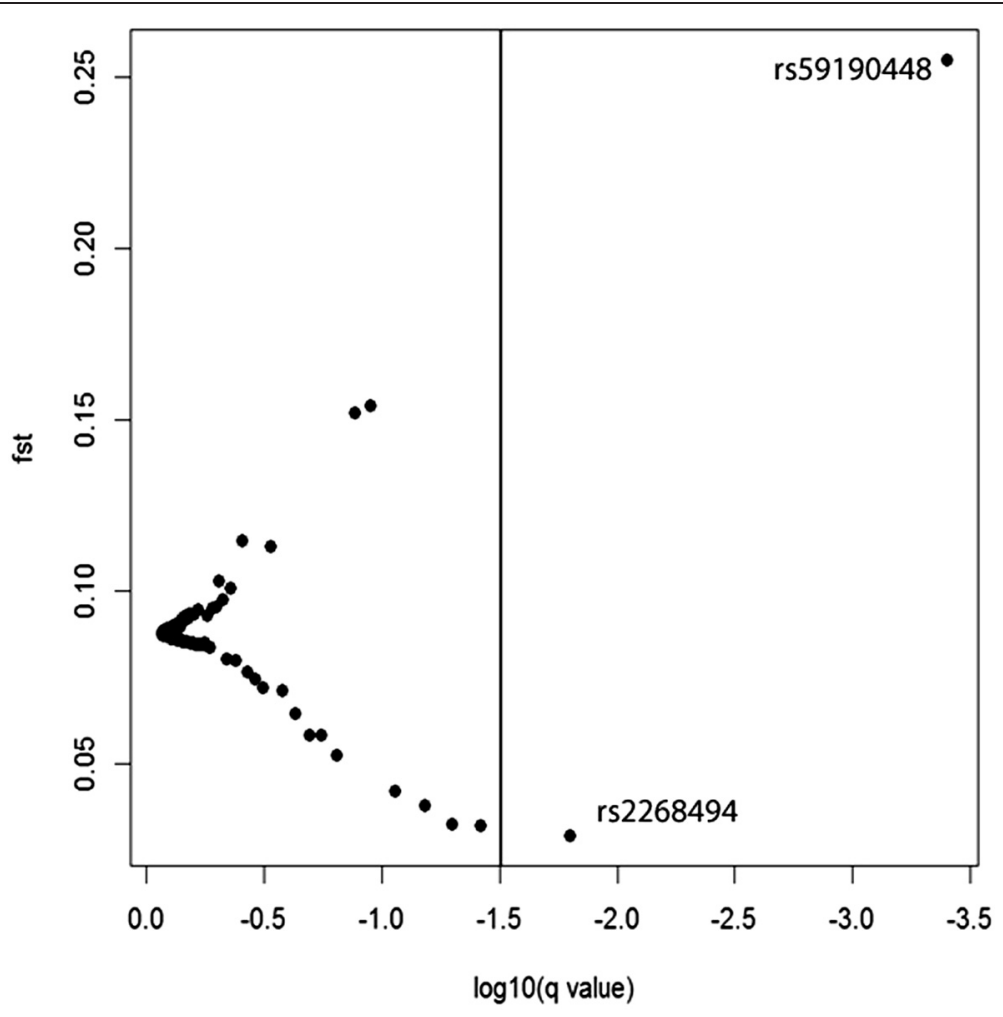

Fig. 3 Detection of outlier SNPs in the OXTR gene using BayeScan. BayeScan indicates 'very strong' evidence for selection (the vertical line corresponds to $\log 10(\mathrm{BF})>1.5$ ) for the two loci rs59190448 and rs2268494. Log 10(q value) are shown on the x-axis and FST-values on the y axis. FST: locus-specific genetic divergence among human populations. Log10(q-value): decision factor in logarithmic scale (base 10) to determine selection

transcriptional repressor that repress neuronal genes in non-neuronal tissues. It remains unknown whether this SNP influences the expression of this gene. Nonetheless, as we had access to SNP data only from 1000 Genomes, we were unable to include the repeat regions in the promotor

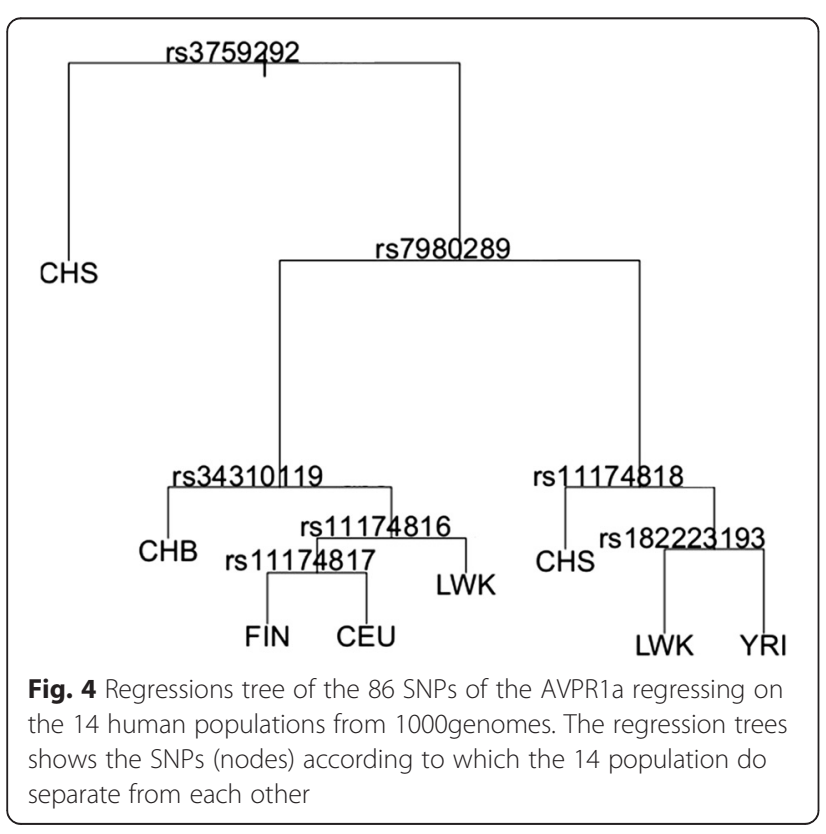

of AVPR1a, previously identified as being related to social behaviour, in our analysis. This prohibits drawing definitive conclusions about a particularly directional selection of AVPR1a (e. g., because we think that the SNP detected as being under positive selection should be treated with caution).

Besides balancing selection, we also found strong evidence of positive directional selection. This was detected by all three used approaches in the intron 3 of the OXTR gene (SNP rs59190448), indicating adaptive evolution at this locus. Very recently, Myers et al. [48] found evidence that rs59190448 modulates the relationship between early life stress and the risk of anxiety later in life. Additionally, rs59190448 is in high LD (>0.8) with an array of SNPs of known social behavioural associations including: mindreading, empathy $([49,50]$, rs13316193), psychopathy ([16], rs1042778, rs2254298), autism spectrum disorder ([45], rs237889; [9], rs2254298), ADHD ([51], rs13316193), ([52], rs2254298) and pro-social behaviour in cooperation games ([32], rs1042778).

Interestingly a comparison among primate species reveals that, in humans, the reference allele of rs59190448 is $\mathrm{G}$, but $\mathrm{A}$ in the chimp, orang-utan and the macaque. Among these latter species, rs59190448seems to be in an 
Table 2 Identified outlier loci in the AVPR1A gene that are under balancing selection and directional selection using the approaches FDIST, BayeScan and F.LK

\begin{tabular}{|c|c|c|c|c|c|c|c|c|c|c|}
\hline \multirow[b]{2}{*}{ Locus } & \multirow[b]{2}{*}{ Function } & \multicolumn{3}{|l|}{ FDIST } & \multicolumn{3}{|l|}{ BayeScan } & \multicolumn{3}{|c|}{ FLK } \\
\hline & & $H_{E}$ & $F_{S T}$ & $P$ & $\log 10(B F)$ & $q$ & $a_{i}$ & $F_{S T}$ & F.LK & F.LK.p.val \\
\hline rs12830859 & Downstream & 0.2631 & 0.0143 & 0.0000 & 1.1382 & 0.0455 & -1.2413 & 0.0271 & & \\
\hline rs1587098 & Downstream & 0.1412 & 0.0111 & 0.0000 & & & & & & \\
\hline rs1580705 & Downstream & 0.1311 & 0.0176 & 0.0008 & & & & & & \\
\hline rs11174805 & Downstream & 0.1412 & 0.0111 & 0.0000 & & & & & & \\
\hline rs11174806 & Downstream & 0.1412 & 0.0111 & 0.0000 & & & & & & \\
\hline rs11174807 & Downstream & 0.1431 & 0.0124 & 0.0000 & & & & & & \\
\hline rs117072097 & 3'UTR & 0.0272 & 0.0041 & 0.0013 & & & & & & \\
\hline rs11174811 & $3^{\prime} \mathrm{UTR}$ & 0.1467 & 0.0047 & 0.0000 & 1.2742 & 0.0336 & -1.3077 & 0.0255 & & \\
\hline rs34462214 & Intron & 0.1549 & 0.0048 & 0.0000 & $1.5306^{*}$ & 0.0252 & -1.3346 & 0.0248 & & \\
\hline rs3021529 & $5^{\prime} U T R$ & 0.1567 & 0.0057 & 0.0001 & 1.1260 & 0.0270 & -1.2497 & 0.0270 & & \\
\hline rs3759292 & Promoter & & & & & & & & 14.1 & 0.001 \\
\hline rs7308855 & Upstream & 0.1114 & 0.0020 & 0.0000 & $2.1158^{*}$ & 0.0076 & -1.6966 & 0.0178 & & \\
\hline
\end{tabular}

The results with significance $P$ value $<0.005$ are shown for FDIST and F.LK; * the BayeScan log $10(B F)>1.5$ indicating 'very strong' evidence $(P(a \neq .5=0.03 \rightarrow 0.01)$ for selection. All SNPs except SNP rs3759292 are under balancing selection

area of accelerated evolution ([53] - http://genome-euro. ucsc.edu/cgi-bin/hgTracks?db=hg19\&position=chr3\% 3A8802751-8803251\&hgsid=207867630_3B0oNUMNn 7N4rYbLDRmof2xHeoqR).

The regression tree of the genotype regressing on the ethnical affiliation for the OXTR gene may hint at actual population differentiation. The SNP rs59190448 splits the regression tree at its root into the African populations LWK and YRI on one branch and all the other populations on the other branches. The branch with the other non-African populations is consecutively split, separating the two Chinese populations ( $\mathrm{CHS}, \mathrm{CHB}$ ) from the others, by rs9840864, recently found to be associated with reactions to betrayal [44]. This is followed by the

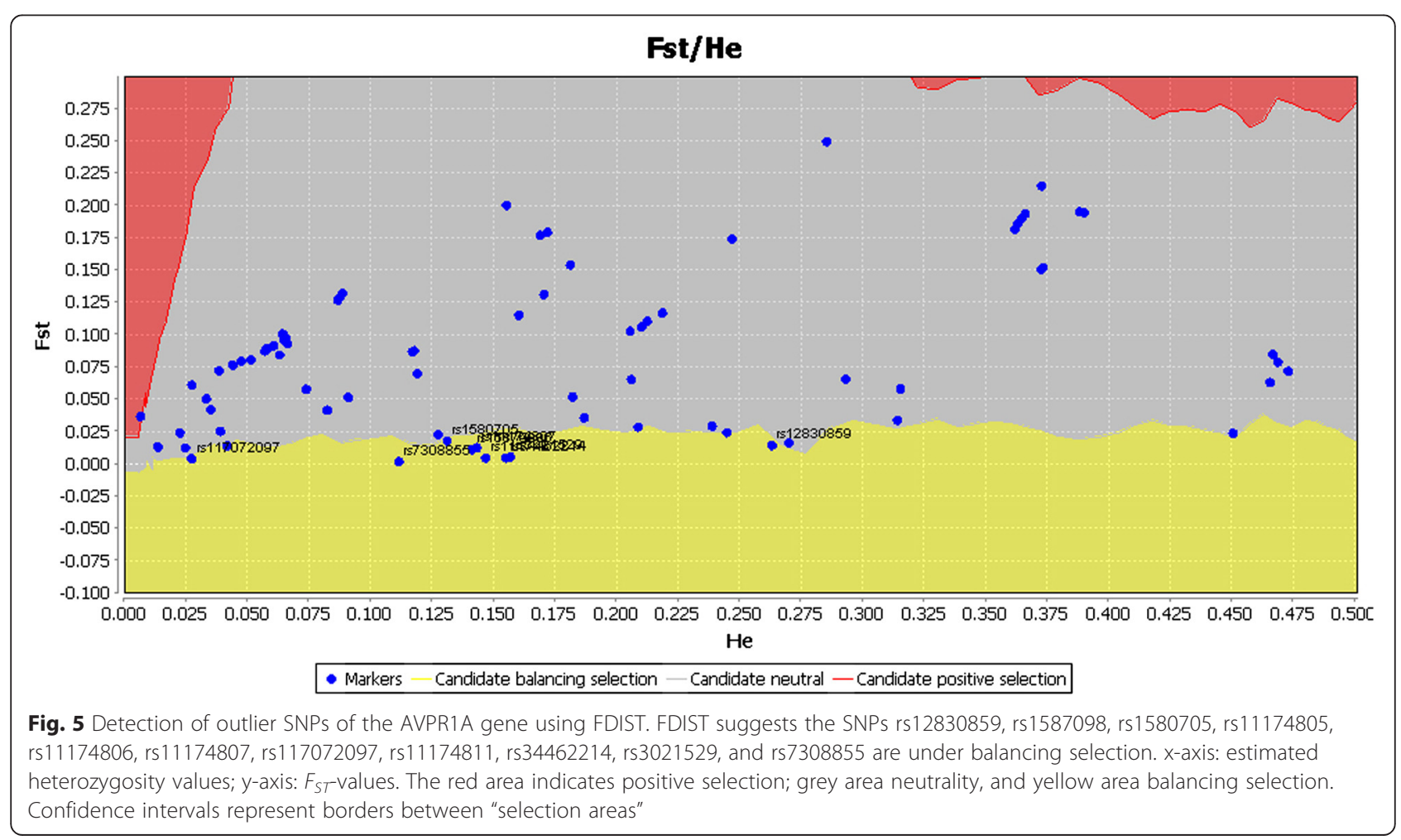




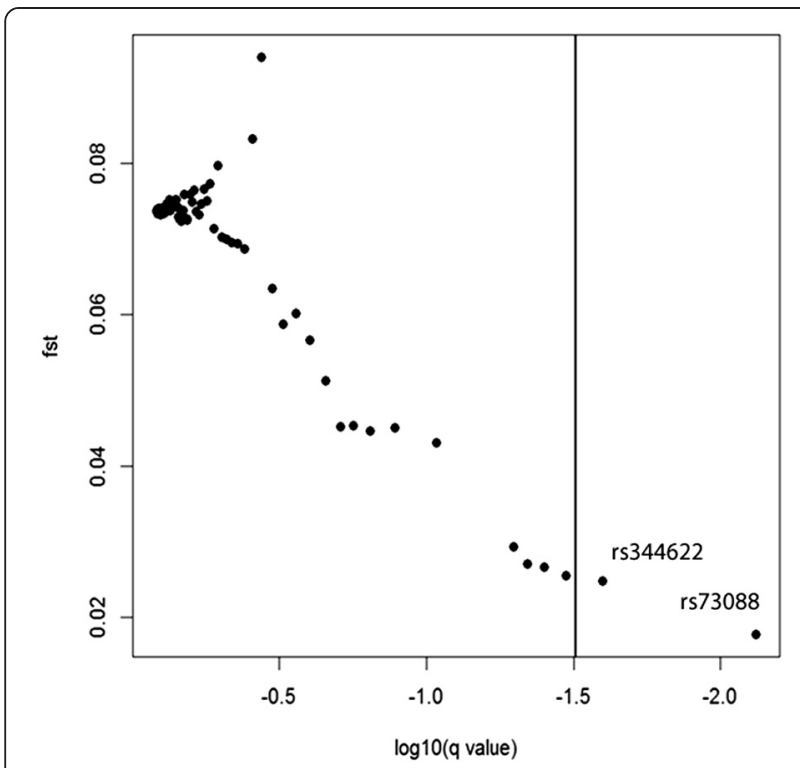

Fig. 6 Detection of outlier SNPs in the AVPR1A gene using BayeScan. BayeScan indicates 'very strong' evidence for selection (the vertical line corresponds to $\log 10(\mathrm{BF})>1.5$ ) for the two loci rs344622 and rs7308855. Log 10(q value) are shown on the x-axis, $F_{S T}$-values on the y axis. $F_{S T}$ : locus-specific genetic divergence among human populations. Log10(q-value): decision factor in logarithmic scale (base 10) to determine selection

Japanese population (JPT), then the European populations (CEU, FIN) and the Mexican population (MXL) suggesting a further differentiation into populations (Fig. 1) according to allele frequencies. The question now is whether the branching is reflected in population differences in behaviour. Although the direct link between behaviour and variation in the OXTR gene is still missing, one hint might come from population differences in human socio-spatial behaviour: interpersonal proximity and touch varies between so-called contact cultures [e.g., Arabs, Latin Americans and Southern Europeans - proximity in descending order] and non-contact cultures [e.g., Asians and Northern Europeans - proximity in descending order] (see [54] for a review).

Although we found no signs of positive selection for the intronic SNP rs53576, we did find that it is in LD' at 0.9696 with rs59190448 in Europeans, and in LD' at 0.959 with rs2324728 and rs237884 in East Asians. And indeed, rs53576 on OXTR has been identified as having a different allelic association depending on the cultural context. This was already exemplified in the introduction section: according to [28], the emotionally sensitive $\mathrm{G}$ allele carriers seek more social support than the AA genotypes, but only in European Americans. In East Asians, in contrast, where emotional support seeking is not the cultural norm, this behaviour is not related to rs53576. Kim et al. [28] further found that different alleles of rs53576 are differently associated with emotional suppression in European Americans and Koreans: among Koreans, emotional suppression was associated with the rs53576 GG genotype, which is characterized as more socio-emotionally sensitive, but not with the AA genotype. In contrast, Americans with the GG genotype were less engaged in emotional suppression compared to those with the AA genotype. Ethnic differences between East Asians and Europeans have also been found for an autistic spectrum disorders risk allele on OXTR (rs2254298; reviewed in [1] and [18]). Finally, oxytocin influences the inter-individual distance between men and women. In an experiment including only female participants, oxytocin-treated women significantly reduced the "comfortable distance" to a male experimenter but not to a female experimenter [55]. Based on this result, it could be speculated - and tested by future studies - that the differences on the OXTR gene also contribute to the known cultural differences in body contact and individual space [56,57]. Nonetheless, these cultural differences do not preclude that second-generation immigrants adopt local norms if they are well-integrated into their host societies.

Additionally, differences in OXTR alleles could result in different regulation of social behaviours and emotions among diverse human cultures. For example, facial expressions are important cues used in social interactions and interpretations of emotional states [58]. Interestingly, the perception of facial signals is not universal among human societies [59]. Jack et al. [60] found differences in the organization of the conceptual space of emotions between East Asians and Western Caucasians. Interestingly, a very recent study showed, for OXTR, a relationship to emotional empathy, whereas AVPR1a was associated with cognitive empathy [61]. Accordingly, our results suggest that molecular biological differences in OXTR alleles may also be related to different perception or cognition of emotions among different socioecologically adapted societies. We may therefore speculate that directional selection processes on OXTR are also associated with the ability to perceive different emotional states (e.g., expressed as facial signals) in diverse human societies. The cognitive part of emotional perception mediated via AVPR1a, however, could be universal for all human societies. As noted above, conclusions on selection on AVPR1a might be limited because the repetitive sequences in the promotor region were not included in our analysis.

Cultural differences are also found in ASD, which is associated with a preference for greater inter-individual distance [62]. ASD has a lower prevalence in Afro-American children than in children with Caucasian ancestry $[63,64]$. 
Nonetheless, any conclusions that ethnic differences in ASD are associated with selection on the OXTR gene would be premature: despite several SNPs on OXTR having been identified as being associated with ASD (reviewed in [22]), the association of OXTR with ASD is weaker than the association of ASD with other genes. The Simons Research Foundation for Autism Research (http://sfari.org/) identified 284 human genes associated with ASD. Compared with other genes involved in ASD, OXTR scores only with "suggestive evidence" (scoring data and scoring methods explained at: https://gene.sfari.org/autdb/GS_Statistics.do). At least in African populations, however, rs59190448 is in high LD' (>0.8) with rs2254298, the SNP, for which an association with autism has been primarily described by $\mathrm{Wu}$ et al. [17] and confirmed in a metaanalysis by [22].

Of special interest are our results of the positive directional selection of one SNP located in intron 3 and the balancing selection in cis-regulative sequences in the 1receptor. These selection processes might reflect adaptations to diverse climate environments or climate changes per se. Darwin noted the challenges of climate changes for the behaviour and survivorship in human evolution in relation to natural selection and compared theses challenges with other animals [65]. Such climate changes have a tremendous influence on terrestrial habitats and therefore on resource availability and body physiology [66,67]. While major genetic changes in body metabolism could have lethal results, behavioural changes for better adaptation to survive in different environments seem to be less risky. Our results could reflect such adaptation processes in humans living under different environmental conditions, i.e., signatures of balancing selection stabilize metabolic processes, and directional selection processes enable these populations to develop adaptive behavioural strategies. Both processes would improve survivorship and reproductive success.

We used very stringent criteria to test for positive selection in the present study to minimise false positives. We might therefore actually underestimate the level of positive selection acting on regulative sequences in both genes. Indeed, a recent study suggests that balancing selection, as a natural consequence of frequent adaptation, is a crucial force maintaining genetic variation [68]. Many functionally important sequence variants are located in this cis-regulatory sequences, playing an important role in the evolution of species-specific expression of genes, and very likely also in individual-specific variation within species. Transcription factor binding sites and other cis-regulatory sequences can be particularly important in the evolutionary adaptation of humans. This is because regulatory elements apparently contribute substantially to both adaptive substitutions and deleterious polymorphisms, with key implications for human evolution and disease [69]. Adaptation-driven balanced polymorphisms could therefore be an important source of consequential genetic variation at genes involved in social cognition.

Balancing selection could be particularly beneficial in fast-changing environments in which diverse human social behaviour patterns might provide an evolutionary adaptive advantage. However, certain variants under selection are linked to detrimental behaviours in humans. These genes with their pleotropic function may therefore maintain balanced polymorphisms that become deleterious in a certain environmental context. The identification of specific positive selection forces on SNPs relevant to gene expression and social behaviour is a promising novel direction for formulating hypotheses about driving forces and constraints in the evolution of human behaviour and social bonding. Likewise, this new approach can provide explanations and context-dependent predictions about within- and between-culture variation in non-verbal behaviour such as interpersonal proximity.

To date, research has strongly concentrated on identifying the genetic basis of social cognition, behaviour, and mental diseases on an individual basis. We assume that the evidence that particularly positive selection has been acting at a SNP on the OXTR gene highlights the importance of a "population perspective". Here, differences between populations due to evolutionary processes should be kept in mind when investigating the genetic predisposition of human behaviour, cognition, and mental diseases. A hint about such differences could be the culturally dependent effects of rs53576 and rs2254298 on emotional support seeking, empathy, and ASD [22,28], both in high LD' with rs59190448. This call for taking potential selection pressures during the relatively recent evolution of Homo sapiens into account.

More generally, the detection of selection in human genes relevant to behaviour could be a starting point for investigating phenotypical associations. This would involve searching for behavioural and cognitive associations with SNPs detected as being under selection as well as SNPs being in high LD' with a SNP under selection.

\section{Conclusions}

This study investigates - based on 1000 Genomes data and using three established methods of selection detection - if a positive selection pressure has been acting on the oxytocin receptor gene (OXTR) and vasopressin receptor gene (AVPR1a). Only for one SNP on the OXTR gene (rs59190448) was a sign of positive directional selection detected with all three methods of selection detection. For rs59190448, however, only one phenotypical association is known, but rs59190448 is in high LD' $(>0.8)$, with variants associated with important pro-social behaviours and mental disorders in humans. We also 
detected various signatures of balancing selection on both neuroreceptor genes. Possible causes for these findings during the evolution and migration of Homo sapiens are discussed, ranging from climate adaptations to social behaviour.

\section{Methods}

\section{Genetic dataset}

The UCSC Genome Browser database/data (http:/genome. ucsc.edu/) were used to retrieve genomic data. The OXTR gene, located on chromosome 3 (p25.3), spans approximately 19 kilobases $(\mathrm{kb})$ and comprises four exons and three introns. Exons 1 and 2 correspond to the $5^{\prime}$ untranslated region (5' UTR), followed by exons 3 and 4 encoding the 389 amino acids of the receptor; intron 3 , which is the largest at $12 \mathrm{~kb}$, separates the coding region immediately after the sixth transmembrane-spanning domain, encoded by exon 3. Exon 4 encodes the seventh transmembrane domain, the $\mathrm{C}$ terminus, and the entire $3^{\prime}$ untranslated region (3' UTR) [70]. The AVPR1A gene is located on chromosome 12 (q14.2) and spans approximately $10 \mathrm{~kb}$, comprising 2 exons; both exons encode the 418 amino acids of the receptor. In this gene, a single intron is located between the exons which encode the sixth and the seventh transmembrane domains [71]. We included single nucleotide polymorphism (SNP) data derived from the 1000 Genomes database (http://www.1000genomes.org/) of 14 human populations from Africa, Asia, Europe, and America (for population abbreviations and studied samples per population see Additional file 1: Table S3), with a minor allele frequency (MAF) of $>0.05$ from the human populations listed in Additional file 1: Table S3, totalling 122 SNPs and 86 SNPS across the entire OXTR gene and AVPR1A gene, respectively. We included about $5 \mathrm{~kb}$ upstream (including promoter regions) and $2 \mathrm{~kb}$ downstream sequences of the coding sequences (genomic position in accordance to the reference genome GRCh37/hg19 on UCSC Genome Browser: OXTR: chr3:8,787,095-8,813,300; AVPR1A: chr12: 63,531,539-63,548,590). We retrieved the SNP data from the 1000 Genome database using Engine SPSmart v5.1.1 (http://spsmart.cesga.es/engines.php?dataSet=engines) [72]. Each genotype was encoded as diploid nucleotides. For all following analyses, we used the population abbreviations according to 1000 Genomes (see Additional file 1: Table S3). We decided to include a small number of base pairs upstream and downstream of the coding region of the genes in order to include only cis- regulative acting sequences such as promoters and enhancer sequences.

\section{Genetic statistical analyses and tests for selection}

We performed the following analyses:

A) To identify the SNPs separating the 14 analysed populations, we calculated a regressing tree of the SNPs regressing on the 14 population identifiers using the $\mathrm{R}$ library $\mathrm{r}$-part ( $\mathrm{R}$ version 3.0.1) [73].

B) We used two different $F_{S T}$-based approaches to detect loci with a pattern deviating from neutrality. Loci that are under balancing selection are expected to show unusually low levels of genetic differentiation (i.e., $F_{S T}<0.05$ ), while loci under directional selection are expected to show unusually high levels of differentiation (i.e., $F_{S T}>0.25$ ) among populations. Based on the analysed 14 human populations $(n=1092)$ from the 1000 Genomes database, we aimed to identify the SNPs under selection by employing the two different $F_{S T}$-based methods: FDIST [74] and BayeScan [75]. Firstly, FDIST calculations were done in the programme LOSITAN (http://popgen.net/soft/lositan/) [76]. LOSITAN evaluates the relationship between $F_{S T}$ and expected heterozygosity $\left(H_{E}\right)$, describing the expected distribution of Wright's inbreeding coefficient $F_{S T}$ vs. $H_{E}$ under an island model of migration with neutral markers; this distribution is used to identify outlier loci that have excessively high or low $F_{S T}$ compared to neutral expectations. In this study, we simulated the neutral distribution of $F_{S T}$ with 100,000 iterations at a significance $P$ value $<0.005$ ( $P$ (Simulation $F_{S T}<$ sample $\left.F_{S T}\right)$; runs were performed using the infinite allele model. LOSITAN also implements a multiple testing correction based on false discovery rates (FDR) to avoid overestimating the percentage of outlier loci. Secondly, we used the programme BayeScan 2.1 (http://population-specific component $\left(\beta_{i}\right)$ shared by all loci, and a locus-specific component $\left(\alpha_{i}\right)$ shared by all the populations, using a logistic regression. Significantly positive values of $\alpha_{i}$ suggest directional selection, and significantly negative values suggest balancing selection (or purifying selection) (for details see [75]). The respective posterior probabilities of these two models are estimated using a reversible-jump Markov Chain Monte Carlo (MCMC) approach. The posterior probability that a locus is subjected to selection $\left(\mathrm{P}\left(\alpha_{i} \neq 0\right)\right)$ is then estimated from the MCMC output by counting the number of times $\alpha_{i}$ is included in the model. Each Markov chain was run for 100,000 updating steps, after 20 pilot runs of 10,000 iterations each and an initial burn-in of $1,000,000$ steps. Samples were collected every 50 steps (thinning). The programme defines a q-value, which is the FDR analogue of the $P$ value. In the present study we chose as the criterion a $\log 10$ Bayes Factor (BF) as the FDR Jeffreys' scale of evidence for Bayes factors = "very strong"which corresponds to a range of q-values of 0.03-0.01, providing very strong evidence that a locus is under selection (programme manual: http://cmpg.unibe.ch/software/ bayescan/). The BayeScan programme provides an R script which was used to control for the FDR and to generate the graphical outputs of the BayeScan results. Additionally, because the error rate of the $F_{S T}$ outlier detection in Lositan and BayeScan has been criticized recently [77], we included an additional approach to verify the SNPs detected as being 
under positive selection by FDIST and BayeScan, the "extended Lewontin and Krakauer test" (FLK). This test compares patterns of differences between allele frequencies in several populations to their expectation under neutral evolution. The null hypothesis of neutral evolution assumes a tree structure with branch length corresponding to the amount of genetic drift in each population (F). The tree is estimated from the matrix of Reynold's genetic distances between populations, using the neighbour joining (NJ) algorithm (for details see [78]); the approach is implemented in the FLK programme, which can be found at https://qgsp.jouy.inra.fr/index.php?option=com_content \&view=article\&id=50\&Itemid=55). As FLK uses the calculated allele frequencies, we included the allele frequencies provided by Engine SPSmart v5.1.1 based on the "1000genome continents" (AFRICA, EUROPE, ASIA and AMERICA) only; therefore, the number of SNPs MAF $>0.05$ included in FLK differs from the number of SNPs included in Lositan and BayeScan (Additional file 1: Table S4 and Additional file 1: Table S5).

C) We further investigated whether any SNPs identified as being under selection are known to influence a phenotypic trait, or whether SNPs known to influence phenotypic traits are in linkage disequilibrium (LD) with the SNPs identified as being under selection (LD threshold $>0.8$ ).

\section{Additional file}

\section{Additional file 1: Includes the following supplementary tables:}

Table S1. FST values, FDIST and BayeScan results for each tested loci of the OXTR gene. Table S2. FST values, FDIST and BayeScan results for each tested loci of the AVPR1a gene. Table S3. Population and sample size used in the study. Table S4. Results of the Lewontin and Krakauer test extended for the SNPs on the OXTR gene. Table S5. Results of the Lewontin and Krakauer test extended for the SNPS on the AVPR1a gene.

\section{Abbreviations}

OXTR: Oxytocin receptor; AVPR1A: Arginine vasopressin receptor 1a; SNP: Single nucleotide polymorphism; LD: Linkage disequilibrium; $5^{\prime}$ UTR: $5^{\prime}$ upstream region; DRD4 7R allele: Seven-repeat variant of the dopamine receptor gene D4; ADHD: Attention-deficit hyperactivity disorder; MAO-A: Monoamine oxidase A

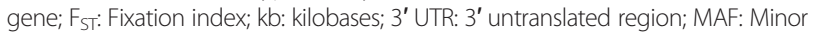
allele frequency; $H E$ : Expected heterozygosity; FDR: False discovery rate; MCMC: Markov Chain Monte Carlo; BF: Bayes Factor; FLK: Extended Lewontin and Krakauer test; NJ: Neighbour joining.

\section{Competing interests}

The authors declare that they have no competing interests.

\section{Authors' contributions}

HS and MF conceived and designed the study, and analysed the data. HS, $\mathrm{SH}, \mathrm{KS}, \mathrm{SW}, \mathrm{BW}$ and $\mathrm{MF}$ did the literature research, wrote and edited the manuscript. HS, SH, KS, SW, BW and MF did participate in the revision of the manuscript. All authors read and approved the final version of the manuscript.

\section{Acknowledgments}

The R Core Team for the statistical computing environment, the 1000 Genomes Project, as well as LOSITAN, BAYESCAN, and FLK. Furthermore, we thank Michael Stachowitsch and the anonymous reviewers for valuable comments on the manuscript. We would also like to thank Horst Seidler for his continuing support. This work was supported by Young Investigator Award 2013 University of Vienna, Faculty of Life Sciences, to SW; Emerging Field "Comparative Human Life History: A Multilevel Approach", University of Vienna, Faculty of Life Sciences, to KS; Back-to-Research Grant, University of Vienna, to SH; and Investitionsprojekt IP 547011, University of Vienna, to MF. The funders had no role in study design, data collection and analysis, decision to publish, or manuscript preparation. We thank the anonymous reviewers for their very comments that significantly improved the manuscript.

\section{Author details}

'Department of Anthropology, University of Vienna, Vienna, Austria.

${ }^{2}$ Cognitive Science Platform at the University of Vienna, Vienna, Austria.

${ }^{3}$ Department of Behavioural Biology, University of Vienna, Vienna, Austria.

Received: 7 November 2014 Accepted: 29 April 2015

Published online: 13 May 2015

\section{References}

1. Ebstein RP, Knafo A, Mankuta D, Chew SH, Lai PS. The contributions of oxytocin and vasopressin pathway genes to human behavior. Horm Behav. 2012;61:359-79.

2. Insel TR. The challenge of translation in social neuroscience: a review of oxytocin, vasopressin, and affiliative behavior. Neuron. 2010;65:768-79.

3. Goodson JL, Thompson RR. Nonapeptide mechanisms of social cognition, behavior and species-specific social systems. Curr Opin Neurobiol. 2010;20:784-94.

4. Thompson RR, George K, Walton JC, Orr SP, Benson J. Sex-specific influences of vasopressin on human social communication. Proc Natl Acad Sci U S A. 2006:103:7889-94.

5. Veenema AH, Bredewold R, De Vries GJ. Sex-specific modulation of juvenile social play by vasopressin. Psychoneuroendocrinology. 2013;38:2554-61.

6. Heinrichs M, Domes $\mathrm{G}$. Neuropeptides and social behaviour: effects of oxytocin and vasopressin in humans. Prog Brain Res. 2008;170:337-50.

7. Goodson JL, Bassn AH. Social behavior functions and related anatomical characteristics of vasotocin/vasopressin systems in vertebrates. Brain Res Rev. 2001;35:246-65.

8. Thompson R, Gupta S, Miller K, Mills S, Orr S. The effects of vasopressin on human facial responses related to social communication.

Psychoneuroendocrinology. 2004;29:35-48.

9. Walum H, Westberg L, Henningsson S, Neiderhiser JM, Reiss D, lgl W, et al Genetic variation in the vasopressin receptor la gene (AVPR1A) associates with pair-bonding behavior in humans. Proc Natl Acad Sci U S A. 2008;105:14153-6.

10. Hopkins WD, Donaldson ZR, Young LJ. A polymorphic indel containing the RS3 microsatellite in the $5^{\prime}$ flanking region of the vasopressin V1a receptor gene is associated with chimpanzee (Pan troglodytes) personality. Gen Brain Behav. 2012;11:552-8.

11. Meyer-Lindenberg A, Kolachana B, Gold B, Olsh A, Nicodemus KK, Mattay V, et al. Genetic variants in AVPR1A linked to autism predict amygdala activation and personality traits in healthy humans. Mol Psychiatry. 2009;14:968-75

12. Kim SJ, Young LJ, Gonen D, Veenstra-Vander Weele J, Courchesne R,

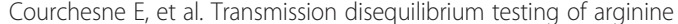
vasopressin receptor 1A (AVPR1A) polymorphisms in autism. Mol Psychiatry. 2002; 7:503-7.

13. Yang SY, Cho S-C, Yoo HJ, Cho IH, Park M, Kim B-N, et al. Association study between single nucleotide polymorphisms in promoter region of AVPR1A and Korean autism spectrum disorders. Neurosci Lett. 2010:479:197-200.

14. Donaldson ZR, Young $\sqcup$. The relative contribution of proximal $5^{\prime}$ flanking sequence and microsatellite variation on brain vasopressin 1a receptor (Avpr1a) gene expression and behavior. Plos Gen. 2013;9:e1003729.

15. Kumsta R, Heinrichs M. Oxytocin, stress and social behavior: neurogenetics of the human oxytocin system. Curr Opin Neurobiol. 2013;23:11-6.

16. Dadds MR, Moul C, Cauchi A, Dobson-Stone C, Hawes DJ, Brennan J, et al. Polymorphisms in the oxytocin receptor gene are associated with the development of psychopathy. Dev Psychopathol. 2014;26:21-31.

17. Wu S, Jia M, Ruan Y, Liu J, Guo Y, Shuang M, et al. Positive association of the oxytocin receptor gene (OXTR) with autism in the Chinese Han population. Biol Psychiatry. 2012;58:74-7. 
18. Liu X, Kawamura Y, Shimada T, Otowa T, Koishi S, Sugiyama T, et al. Association of the oxytocin receptor (OXTR) gene polymorphisms with autism spectrum disorder (ASD) in the Japanese population. J Hum Gen. 2010;55:137-41.

19. Jacob S, Brune CW, Carter CS, Leventhal BL, Lord C, Cook Jr EH. Association of the oxytocin receptor gene (OXTR) in Caucasian children and adolescents with autism. Neurosci Lett. 2007;417:6-9.

20. Campbell DB, Datta D, Jones ST, Lee EB, Sutcliffe JS, Hammock EA, et al. Association of oxytocin receptor (OXTR) gene variants with multiple phenotype domains of autism spectrum disorder. J Neurodev Disord. 2011:3:101-12.

21. Lerer E, Levi S, Salomon S, Darvasi A, Yirmiya N, Ebstein RP. Association between the oxytocin receptor (OXTR) gene and autism: relationship to Vineland Adaptive Behavior Scales and cognition. Mol Psychiatry. 2008;13:980-8

22. LoParo D, Waldman ID: The oxytocin receptor gene (OXTR) is associated with autism spectrum disorder: a meta-analysis. Mol Psychiatry 2014

23. Di Napoli A, Warrier V, Baron-Cohen S, Chakrabarti B. Genetic variation in the oxytocin receptor (OXTR) gene is associated with asperger syndrome. Mol Autism. 2014;5:48.

24. Rodrigues SM, Saslow LR, Garcia N, John OP, Keltner D. Oxytocin receptor genetic variation relates to empathy and stress reactivity in humans. Proc Natl Acad Sci U S A. 2009;106:21437-41.

25. Bakermans-Kranenburg MJ, van IJzendoorn MH. Oxytocin receptor (OXTR) and serotonin transporter $(5-\mathrm{HTT})$ genes associated with observed parenting. Soc Cogn Affect Neurosci. 2008;3:128-34.

26. Feldman R, Zagoory-Sharon O, Weisman O, Schneiderman I, Gordon I, Maoz R, et al. Sensitive parenting is associated with plasma oxytocin and polymorphisms in the OXTR and CD38 genes. Biol Psychiatry. 2012;72:175-81.

27. Walum H, Lichtenstein P, Neiderhiser JM, Reiss D, Ganiban JM, Spotts EL, et al. Variation in the oxytocin receptor gene is associated with pair-bonding and social behavior. Biol Psychiatry. 2012;71:419-26.

28. Kim HS, Sherman DK, Mojaverian T, Sasaki JY, Park J, Suh EM, et al. Gene-culture interaction: oxytocin receptor polymorphism (OXTR) and emotion regulation. Soc Psychol Pers Sci. 2011;2:665-72.

29. Kogan A, Saslow LR, Impett EA, Oveis C, Keltner D, Saturn SR. Thin-slicing study of the oxytocin receptor (OXTR) gene and the evaluation and expression of the prosocial disposition. Proc Natl Acad Sci U S A. 2011;108:19189-92.

30. Krueger F, Parasuraman R, lyengar $V$, Thornburg M, Weel J, Lin M, et al. Oxytocin receptor genetic variation promotes human trust behavior. Front Hum Neurosci. 2012;6:4.

31. Lucht MJ, Barnow S, Sonnenfeld C, Ulrich I, Grabe HJ, Schroeder W, et al. Associations between the oxytocin receptor gene (OXTR) and "mind-reading" in humans-an exploratory study. Nord J Psychiatry. 2013;67:15-21.

32. Israel S, Lerer E, Shalev I, Uzefovsky F, Riebold M, Laiba E, et al. The oxytocin receptor (OXTR) contributes to prosocial fund allocations in the dictator game and the social value orientations task. Plos One. 2009;4:e5535.

33. Westberg L., Hasse Walum H: Oxytocin and Vasopressin Gene Variation and the Neural Basis of Social Behaviors. The Oxford Handbook of Molecular Psychology 2015. DOl: 10.1093/oxfordhb/9780199753888.013.011

34. Vallender EJ, Lahn BT. Positive selection on the human genome. Hum Mol Gen. 2004;13:R245-54.

35. Ding YC, Chi HC, Grady DL, Morishima A, Kidd JR, Kidd KK, et al. Evidence of positive selection acting at the human dopamine receptor D4 gene locus. Proc Natl Acad Sci U S A. 2002;99:309-14.

36. Naka I, Nishida N, Ohashi J. No evidence for strong recent positive selection favoring the 7 repeat allele of VNTR in the DRD4 gene. Plos One. 2011;6:e24410.

37. Bhaduri N, Das M, Sinha S, Chattopadhyay A, Gangopadhyay PK, Chaudhur $\mathrm{K}$, et al. Association of dopamine D4 receptor (DRD4) polymorphisms with attention deficit hyperactivity disorder in Indian population. Am J Med Gen B -Neuropsychiat Gen. 2006;141B:61-6.

38. Faraone SV, Doyle AE, Mick E, Biederman J. Meta-analysis of the association between the 7-repeat allele of the dopamine D-4 receptor gene and attention deficit hyperactivity disorder. Am J Psychiatry. 2001;158:1052-7.

39. Gilad Y, Rosenberg S, Przeworski M, Lancet D, Skorecki K. Evidence for positive selection and population structure at the human MAO-A gene. Proc Natl Acad Sci U S A. 2002;99:862-7.

40. Brunner HG, Nelen MR, Vanzandvoort P, Abeling N, Vangennip AH, Wolters EC, et al. X-linked borderline mental retardation with prominent behavioural disturbance phenotype, genetic localization, and evidence for disturbed monoamine metabolism. Am J Hum Genet. 1993;52:1032-9.

41. Buckholtz JW, Meyer-Lindenberg A. Psychopathology and the human connectome: toward a transdiagnostic model of risk for mental illness. Neuron. 2012;74:990-1004.

42. Meyer JH, Ginovart N, Boovariwala A, Sagrati S, Hussey D, Garcia A, et al. Elevated monoamine oxidase A levels in the brain - an explanation for the monoamine imbalance of major depression. Arch General Psychiatry. 2006;63:1209-16.

43. Genomes Project Consortium. An integrated map of genetic variation from 1,092 human genomes. Nature. 2012;491:56-65.

44. Tabak BA, McCullough ME, Carver CS, Pedersen EJ, Cuccaro ML. Variation in oxytocin receptor gene (OXTR) polymorphisms is associated with emotional and behavioral reactions to betrayal. Soc Cogn Affect Neurosci. 2014;9:810-6.

45. Iwase S, Xiang B, Ghosh S, Ren T, Lewis PW, Cochrane JC, et al. ATRX ADD domain links an atypical histone methylation recognition mechanism to human mental-retardation syndrome. Nature Struct Mol Biol. 2011;18:769-76.

46. Lynch VJ, Leclerc RD, May G, Wagner GP. Transposon-mediated rewiring of gene regulatory networks contributed to the evolution of pregnancy in mammals. Nat Gen. 2011:43:1154-8.

47. Maher BS, Vladimirov VI, Latendresse SJ, Thiselton DL, McNamee R, Kang M, et al. The AVPR1A gene and substance use disorders: association, replication, and functional evidence. Biol Psychiat. 2011;15:7519-27.

48. Myers AJ, Williams L, Gatt JM, McAuley-Clark EZ, Dobson-Stone C, Schofield $P R$, et al. Variation in the oxytocin receptor gene is associated with increased risk for anxiety, stress and depression in individuals with a history of exposure to early life stress. J Psychiat Res. 2014;59:93-100.

49. Wu N, Li Z, Su Y. The association between oxytocin receptor gene polymorphism (OXTR) and trait empathy. J Affect Disorders. 2012;138:468-72.

50. Schneiderman I, Kanat-Maymon Y, Ebstein RP, Feldman R. Cumulative risk on the oxytocin receptor gene (OXTR) underpins empathic communication difficulties at the first stages of romantic love. Soc Cog Affect Neurosci. 2014;9:1524-9.

51. Park J, Willmott M, Vetuz G, Toye C, Kirley A, Hawi Z, et al. Evidence that genetic variation in the oxytocin receptor (OXTR) gene influences social cognition in ADHD. Prog Neur-Psychoph. 2010;34:697-702.

52. Thompson RJ, Parker KJ, Hallmayer JF, Waugh CE, Gotlib IH. Oxytocin receptor gene polymorphism (rs2254298) interacts with familial risk for psychopathology to predict symptoms of depression and anxiety in adolescent girls. Psychoneuroendocrinology. 2011;36:144-7.

53. Pollard KS, Hubisz MJ, Rosenbloom KR, Siepel A. Detection of nonneutral substitution rates on mammalian phylogenies. Gen Res. 2010;20:110-21.

54. Argyle M: Bodily Communication. Routledge, Kindle edition (2nd edition); 2013

55. Preckel K, Scheele D, Kendrick KM, Maier W, Hurlemann R. Oxytocin facilitates social approach behavior in women. Front Behav Neurosci. 2014;8:191.

56. Dibiase R, Gunnoe A. Gender and culture differences in touching behavior. J Soc Psychol. 2004;144:49-62.

57. Matsumoto D: Culture and nonverbal behaviour. In The Hidden Dimension Edited by Hall ET. Anchor; 1990:219-235.

58. Lischke A, Berger C, Prehn K, Heinrichs M, Herpertz SC, Domes G. Intranasal oxytocin enhances emotion recognition from dynamic facial expressions and leaves eye-gaze unaffected. Psychoneuroendocrinology. 2012;37:475-81

59. Elfenbein HA, Ambady N. On the universality and cultural specificity of emotion recognition: a meta-analysis. Psychol Bull. 2002;128:203-35.

60. Jack RE, Garrod OG, Yu H, Caldara R, Schyns PG. Facial expressions of emotion are not culturally universal. Proc Natl Acad Sci U S A. 2012:8: 109:7241-4.

61. Uzefovsky F, Shalev I, Israel S, Edelman S, Raz Y, Mankuta D, et al. Oxytocin receptor and vasopressin receptor 1a genes are respectively associated with emotional and cognitive empathy. Horm Behav. 2015;67:60-5.

62. Gessaroli E, Santelli E, di Pellegrino G, Frassinetti F. Personal space regulation in childhood autism spectrum disorders. Plos One. 2013;8:e74959.

63. Mandell DS, Ittenbach RF, Levy SE, Pinto-Martin JA. Disparities in diagnoses received prior to a diagnosis of autism spectrum disorder. J Autism Dev Disord. 2007:37:1795-802.

64. Mandell DS, Wiggins LD, Carpenter LA, Daniels J, DiGuiseppi C, Durkin MS, et al. Racial/ethnic disparities in the identification of children with autism spectrum disorders. Am J Public Health. 2009;99:493-8. 
65. Fellmann F, Wallner B, editors. Charles darwin: Die abstammung des menschen und die sexuelle selektion. Stuttgart: Reclam. Fincher CL; 2012

66. Cerling TE, Wynn JG, Andanje SA, Bird MI, Korir DK, Levin NE, et al. Woody cover and hominin environments in the past 6 million years. Nature. 2011;476:51-5.

67. deMenocal PB. African climate change and faunal evolution during the Pliocene-Pleistocene. Earth Planet Sc Lett. 2004;220:3-24.

68. Sellis D, Callahan BJ, Petrov DA, Messer PW. Heterozygote advantage as a natural consequence of adaptation in diploids. Proc Natl Acad Sci U S A. 2011;108:20666-71.

69. Arbiza L, Gronau I, Aksoy BA, Hubisz MJ, Gulko B, Keinan A, et al. Genomewide inference of natural selection on human transcription factor binding sites. Nat Genet. 2013:45:723-9.

70. Inoue T, Kimura T, Azuma C, Inazawa J, Takemura M, Kikuchi T, et al. Structural organization of the human oxytocin receptor gene. J Biol Chem. 1994;269:32451-6.

71. Thibonnier M, Graves MK, Wagner MS, Auzan C, Clauser E, Willard HF. Structure, sequence, expression, and chromosomal localization of the human V-1a vasopressin receptor gene. Genomics. 1996;31:327-34

72. Amigo J, Salas A, Phillips C, Carracedo A. SPSmart: adapting population based SNP genotype databases for fast and comprehensive web access. BMC Bioinformatics. 2008:9:428.

73. R Core Team: R: A Language and Environment for Statistical Computing. R Foundation for Statistical Computing, Vienna, Austria; 2013 [http://www.R-project.org/].

74. Beaumont MA, Nichols RA. Evaluating loci for use in the genetic analysis of population structure. Proc R Soc Lond [Biol]. 1996;263:1619-26.

75. Foll M, Gaggiotti O. A genome-scan method to identify selected loci appropriate for both dominant and codominant markers: A Bayesian perspective. Genetics. 2008;180:977-93.

76. Antao T, Lopes A, Lopes RJ, Beja-Pereira A, Luikart G. LOSITAN: A workbench to detect molecular adaptation based on a F(st)-outlier method. BMC Bioinformatics. 2008:9:323.

77. Lotterhos K, Whitlock MC. Evaluation of demographic history and neutral parametrization of the performance of the Fst outlier tests. Mol Ecol. 2014;23:2178-92.

78. Bonhomme M, Chevalet C, Servin B, Boitard S, Abdallah J, Blott S, et al. Detecting selection in population trees: the Lewontin and Krakauer test extended. Genetics. 2010;186:241-62.

\section{Submit your next manuscript to BioMed Central and take full advantage of:}

- Convenient online submission

- Thorough peer review

- No space constraints or color figure charges

- Immediate publication on acceptance

- Inclusion in PubMed, CAS, Scopus and Google Scholar

- Research which is freely available for redistribution 